\title{
Parotidite supurativa aguda em paciente jovem e sistemicamente saudável
}

\author{
Acute suppurative parotitis in young \\ and systemically healthy patient
}

\author{
MILlena LoRRANA De ALmeIDA SOUSA ${ }^{1}$ \\ JAQUELINE OLIVEIRA BARRETO ${ }^{1}$ \\ MARIA VitóRIa CALAdO RAMALHO dOS SANTOS ${ }^{1}$ \\ GEORGE BORJA DE FREITAS ${ }^{2}$ \\ IRLEY GONZAGa MACHADO PATRIOTA ${ }^{2}$ \\ JULIERME FERREIRA ROCHA ${ }^{1}$
}

\begin{abstract}
RESUMO
Objetivo: Este trabalho teve por objetivo descrever um caso de um paciente jovem e sistemicamente saudável que desenvolveu um quadro de parotidite supurativa aguda. Relato de caso: Paciente do gênero masculino, 21 anos de idade, feoderma, procurou os serviços da Liga Acadêmica de Cirurgia, no Departamento de Cirurgia Bucomaxilofacial do curso de odontologia da Universidade Federal de Campina Grande, campus Patos, Paraíba, Brasil, apresentando como queixa principal aumento de volume e dor na região esquerda da face. No exame físico observou-se drenagem de secreção purulenta pelo ducto de Stensen e o diagnóstico clínico foi de parotidite supurativa aguda. O tratamento indicado foi a base de antibiótico e anti-inflamatório não esteroidal, o paciente evoluiu bem e após sete dias não apresentava qualquer queixa clínica. Conclusão: Esta infeção é incomum em pacientes jovens e saudáveis, mas quando diagnosticada precocemente e tratada de maneira adequada apresenta um bom prognóstico.
\end{abstract}

Palavras-chave: Parotidite, Glândula parótida, Sialodenite.

\begin{abstract}
Objective: To describe a case of a young and systemically healthy patient who developed acute suppurative parotitis. Case report: A 21-year-old male patient, feoderma, sought the services of the Academic League of Surgery at the Department of Bucomaxilofacial Surgery at the Federal University of Campina Grande, Patos campus, Paraíba, Brazil complaining of a increased volume and pain in the left region of the face. On physical examination, a purulent drainage of the Stensen duct was observed and the clinical diagnosis was acute suppurative parotitis. The indicated treatment was based on antibiotic and non-steroidal antiinflammatory, the patient progressed well and after seven days had no clinical complaint. Conclusion: This infection is uncommon in young and healthy patients, but when diagnosed early and properly treated it has a good prognosis.

Keywords: Parotitis, Parotid gland, Sialadenitis.
\end{abstract}

\footnotetext{
${ }^{1}$ Faculdade de Odontologia, UFCG Universidade Federal de Campina Grande, Patos, PB, Brasil

${ }^{2}$ Universidade das Faculdades Integradas de Patos, PB, Brasil
} 


\section{INTRODUçÃo}

A parotidite supurativa aguda (PSA) é um quadro infeccioso de origem bacteriana, que atinge a glândula parótida e afeta principalmente os pacientes que apresentam quadros de xerostomia ou hipossalivação associada a má higiene bucal. ${ }^{1-2} \mathrm{~A}$ diminuição do fluxo salivar geralmente está associada ao uso de medicamentos, desidratação póscirúrgica, síndrome de Sjogren, obstrução do ducto de Stensen ${ }^{3}$ ou por alterações de ordem sistêmica como diabetes e alcoolismo. ${ }^{4}$

A PSA se comporta de forma padronizada pelo aumento de volume repentino, eritematoso e dolorido na face com drenagem de secreção purulenta pelo ducto de Stensen.1,5 0 diagnóstico deve ser estabelecido com base nos achados clínicos associados a exames de imagem como a ultrassonografia ou tomografia computadorizada. ${ }^{6}$

O tratamento da PSAé bem estabelecido na literatura e consiste em medicação antibiótica empírica que pode ser associada a terapias coadjuvantes na tentativa de reverter o quadro clínico sistêmico. Quando não há regressão dos sintomas uma intervenção cirúrgica pode ser necessária..$^{7-9}$

Este trabalho teve por objetivo apresentar um caso de parotidite supurativa aguda em paciente jovem.

\section{Relato de caso}

Paciente do gênero masculino, 21 anos de idade, feoderma, procurou os serviços da Liga Acadêmica de Cirurgia no Departamento de Cirurgia Bucomaxilofacial do curso de odontologia da Universidade Federal de Campina Grande, campus Patos, Paraíba, Brasil, apresentando como queixa principal o aumento de volume repentino e dor na região esquerda da face, trazendo consigo um radiografia panorâmica.
O paciente possuía uma boa condição de saúde geral, sem histórico de doença sistêmica, não fazia uso de qualquer medicação e negou trauma na região. No exame físico foi possível observar um aumento de volume na região da glândula parótida esquerda, causando assimetria facial. A região apresentava coloração semelhante à da pele e consistência endurecida à palpação. O grau de abertura bucal e a função do Nervo facial estavam dentro do padrão de normalidade (Fig. 1A).

No aspecto intrabucal foi observado fluxo salivar regular e higiene oral satisfatória. Ao realizar a massagem e ordenha da glândula parótida esquerda houve a excreção de exsudato purulento pelo ducto de Stensen, um sinal patognomônico para infecção da glândula. Assim, o diagnóstico clínico de parotidite supurativa aguda foi estabelecido (Fig. 1B).

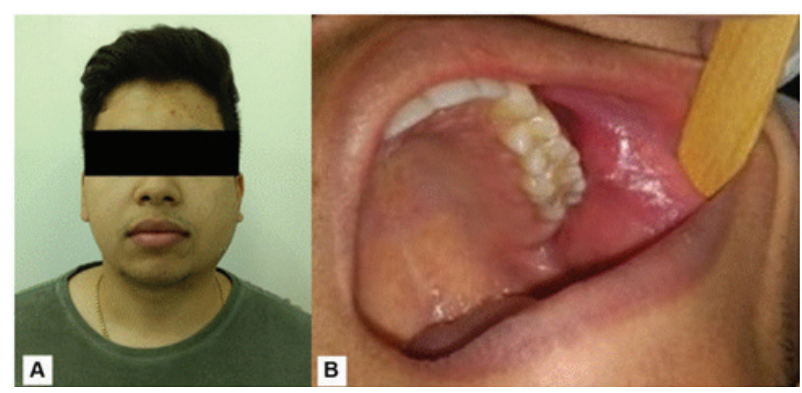

Figura 1 - A. Aspecto clínico inicial; B. Aspecto clínico intra-oral

$\mathrm{Na}$ radiografia panorâmica observou-se estruturas ósseas e dentárias com aspecto de normalidade (Fig. 2A). No entanto, a ultrassonografia evidenciou aumento de volume da glândula envolvida e presença de imagens hipoecogênicas sugestivas de linfonodos intraglandulares em número de, pelo menos, cinco, situados nos lobos superficial e profundo da glândula esquerda, compatível com parotidite (Fig. 2B). 

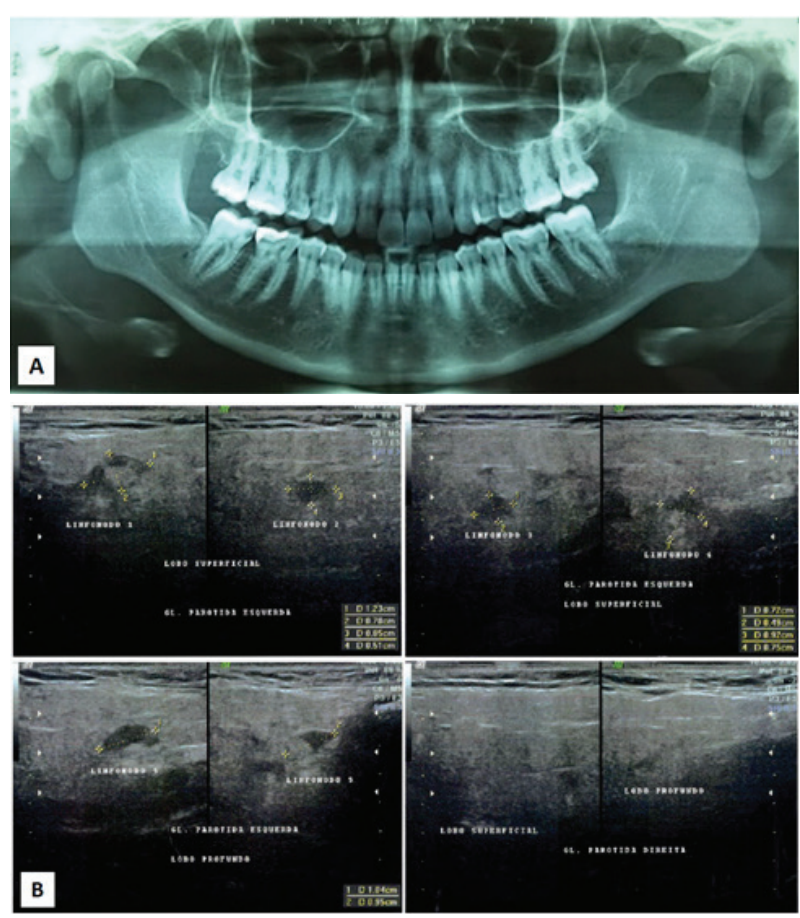

Figura 2 - A. Radiografia panorâmica dos maxilares; B. Ultrassonografia das glândulas parótidas

Iniciou-se a terapia antibiótica (Amoxicilina $875 \mathrm{mg}$ e clavulanato de potássio $125 \mathrm{mg}$, a cada doze horas) durante sete dias, associado a prescrição de anti-inflamatório não esteroidal (Nimesulida 100mg a cada doze horas) durante três dias. O paciente foi instruído a retornar após 24 e 48 horas para reavaliação.

Nas primeiras vinte e quatro horas o edema aumentou em grandes proporções, mas com a continuidade do tratamento medicamentoso, após quarenta e oito horas houve regressão significativa dos sinais clínicos, e no sétimo dia o paciente apresentou-se completamente assintomático (Fig. 3 A e B)

\section{Discussão}

Parotidite supurativa aguda é uma doença bem estabelecida na literatura científica. Porém, o que faz este caso incomum, é o fato do paciente não possuir qualquer manifestação clínica, local ou sistêmica, capaz de predispor o surgimento da infecção, visto que a maior

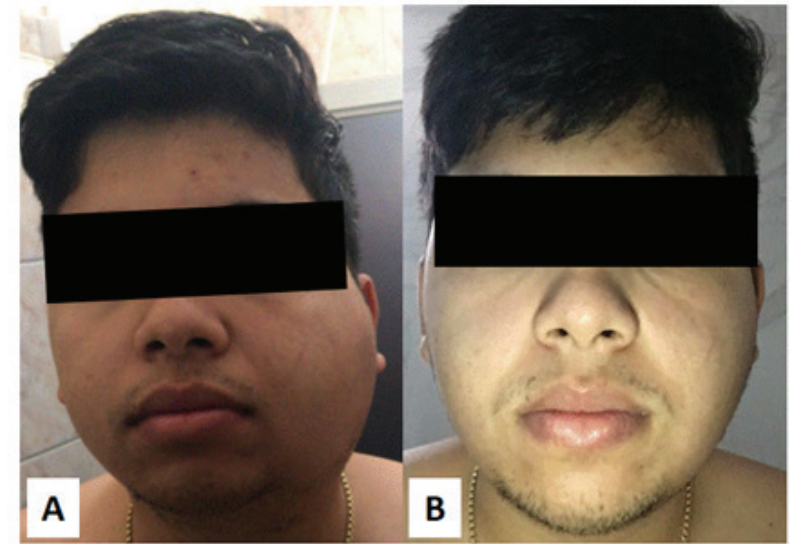

Figura 3 - A. Aspecto clínico após 24 horas. B Aspecto clínico após 48 horas.

parte dos casos estão relacionados a baixa produção de saliva, desidratação pós-cirúrgica, desnutrição, imunossupressão, síndrome de Sjögren e pelo uso de medicamentos como antidepressivos, anticolinérgicos e diuréticos ${ }^{6,8}$ que facilita a propagação de bactérias do meio bucal para o interior da glândula através do ducto de Stensen. ${ }^{1,5,6}$

Contudo, na maioria dos casos publicados a infecção se desenvolveu de forma unilateral como em nosso relato ${ }^{10,11,12,13,14,15}$, embora se encontre relatos de PSA bilateral em pacientes recém nascidos. ${ }^{16,17}$

O diagnóstico deve ser baseado nas informações clínicas e na história relatada pelo paciente. Porém, quando há suspeita de PSA, exames por imagem devem ser realizados para uma avaliação mais precisa da região. O paciente compareceu à clínica para consulta inicial com uma radiografia panorâmica em mãos, porém a mesma não representa um exame adequado para visualização das glândulas, e foi útil apenas para descartar lesões intra-ósseas e infecção de origem dentária. Segundo Zajkowski, (2016) ${ }^{18}$ a ultrassonografia é o melhor método para esses casos, eles afirmam que o aumento de volume da glândula e a presença de linfonodos intraparotídeos podem sugerir uma condição infecciosa, como visto nos achados ecográficos do paciente. 
Outros exames como a tomografia computadorizada e a ressonância magnética nuclear fornecem uma exploração ainda mais precisa da glândula parótida e do tecido circundante, no entanto não são indicados como primeira escolha pelo alto custo. ${ }^{19}$ Assim como, a sialografia com injeção de contraste, que gera bastante desconforto nos casos de infecção aguda, e também não deve ser empregada. ${ }^{6}$

Quando estabelecido o diagnóstico de PSA, Lampropoulos (2012) ${ }^{14}$ aconselham que o clínico avalie a saúde geral do paciente, sua resposta fisiológica e a patogenicidade dos microrganismos. Como visto, o caso reportado apresentava pontos positivos em todos os parâmetros, o que certamente facilitou a condução do caso.

Assim, devido à natureza polimicrobiana da PSA, que na maior parte dos casos - Staphylococcus aureus e as bactérias anaeróbias gram-negativas estão presentes ${ }^{5}$ Fattahi $(2002)^{6}$ indicavam o tratamento à base de penicilina, cefalosporina de primeira geração ou clindamicina. Contudo, Wilson (2014) ${ }^{20}$ afirma que muitos desses microrganismos são resistentes à penicilina pela capacidade de produzir inibidores da beta-lactamase e recomendam que a droga seja associada ao clavulanato de potássio.

Isso se assemelha ao presente caso, tendo em vista que essa foi a antibioticoterapia de escolha, por meio da qual se obteve sucesso do tratamento. Entretanto, não necessitou saber quais microorganismos estavam envolvidos na infecção, pois o paciente apresentava quadro de urgência, o que levou a escolha pelo o tratamento empírico, cuja resposta foi positiva, e não houve necessidade de realizar teste de cultura bacteriana.

Por outro lado, Patel, (2017) ${ }^{11}$ descreveram um caso de parotidite em uma mulher com 50 anos de idade, estado de saúde debilitado, baixo peso, que fazia uso de medicamentos anticolinérgicos e tinha hábitos deletérios (álcool e tabaco), cujo tratamento com amoxicilina e clavulanato não foi eficiente. Isso provavelmente se explica pela diversidade e patogenicidade dos microrganismos presentes na infecção e o comprometimento sistêmico do paciente que pode interferir na resposta fisiológica do organismo.

Além disso, alguns autores defendem que terapias coadjuvantes como reidratação, fisioterapia com calor, instruções de higiene oral e suporte nutricional têm grande contribuição à terapêutica medicamentosa. ${ }^{7,8,9}$. Contudo, existe uma carência de ensaios clínicos atualizados sobre a influência do tratamento farmacológico e das terapias coadjuvantes no curso natural da doença. Provavelmente, deve-se ao fato da dificuldade de obtenção de uma amostra suficiente. No caso descrito, essas medidas não foram necessárias, pois o paciente apresentava condição sistêmica favorável.

É importante também que o clínico esteja apto a diferenciar a parotidite supurativa da não supurativa como a parotidite viral (caxumba), sialolitíase, lesões císticas e neoplasias, que possuem aspecto clínico semelhante e a diferenciação se faz pela presença de secreção no ducto de Stensen, que é um achado clínico presente apenas em infecções bacterianas. ${ }^{21-22}$

Nessa perspectiva, fica claro a importância do diagnóstico adequado e uso correto de antibióticos onde a maioria dos pacientes respondem bem. Entretanto, quando isso não ocorre, a progressão contínua da infecção se dissemina pelos espaços faciais com potencial de desenvolver abscessos e comprometer estruturas anatômicas nobres, cujo manejo cirúrgico para incisão e drenagem se faz necessário, tornando o tratamento mais complexo e invasivo. ${ }^{13,14}$

\section{Conclusão}

O desenvolvimento da PSA é incomum, podendo ocorrer em todas as idades, inclusive 
em pacientes jovens e saudáveis. Mas quando diagnosticada precocementee tratada demaneira adequada apresenta um bom prognóstico.

\section{REFERÊNCIAS}

1. Mandel L. Differentiating acute suppurative parotitis from acute exacerbation of a chronic parotitis: case reports. J Oral Maxillofac Surg. 2008; 66 (9):1964-1968. DOI: 10.1016/j. joms.2007.08.008

2. Matsuo T. Accute supurative parotitis caused by a fish bone: a case report. Int $\mathrm{J}$ Oral Maxillofac Surg. 1997; 26(1): 54. doi: 10.1016/s09015027(97)80849-1.

3. Wahba HN. Sjögren's syndrome and acute suppurative parotitis. J Rheumatol. 1993; 20(2):404. https://pubmed.ncbi.nlm.nih.gov/8474091/

4. Krippaehne WW, Hunt TK, Dunphy JE. Acute suppurative parotitis: a study of 161 cases. Ann Surg. 1962; 156(2): 251-7. doi: 10.1097/00000658-196208000-00010

5. Fattahi TT, Lyu PE, Van Sickels JE. Management of Acute Brook I. Diagnosis and management of parotitis. Arch Otolaryngol Head Neck Surg. 1992; 118 (5):469-71.

6. Suppurative Parotitis. J Oral Maxillofac Surg. 2002; 60(4): 446-8. https://www.scielo.br/pdf/ rimtsp/v50n5/a10v50n5.pdf

7. Andrews JC,Abemayor E, Alessi DM, Canalis RF. Parotitis and facial nerve dysfunction. Arch Otolaryngol Head Neck Surg. 1989; 115(2): 240-2. DOI: 10.1001/archotol.1989.01860260114026

8. Brook I. Acute bacterial suppurative parotitis: microbiology and management. J Craniofac Surg. 2003; 14(1):37-40. DOI: 10.1097/00001665200301000-00006

9. Rousseau P. Acute suppurative parotitis. J Am Geriatr Soc. 1990; 38(8): 897-8. DOI: 10.1111/ j.1532-5415.1990.tb05707.x

10. Avcu G, Belet N, Karli A, Sensoy G. Acute suppurative parotitis in a 33-day-old patient. J Trop Pediatr. 2015; 61(3): 218-21. DOI: 10.1093/tropej/fmv012

11. Patel $P$, Scott $S$, Cunningham S. Challenging Case of Parotitis: A Comprehensive Approach. J Am Osteopath Assoc. 2017; 117(12): 13740. doi:https://doi.org/10.7556/jaoa.2017.152
12. Sellami M, Mnejja M, Masmoudi M, Maalej F, Charfeddine I, Ghorbel A. La parotidite bactérienne aiguë compliquée de l'adulte. Presse Med. 2017; 46 (5): 542-4. DOI : 10.11604/ pamj.2016.24.286.10124

13. Grinnell M, Logeman A, Knudsen T, Sayed Z. Deep Parotid Lobe Abscess Presenting with Dysphagia and Trismus. Case Rep Otolaryngol. 2019; 2019:1-4. doi: 10.1155/2019/2931015

14. Lampropoulos P, Rizos S, Marinis A. Acute Suppurative Parotitis: A Dreadful Complication in Elderly Surgical Patients. Surg Infect. 2012;13(4): 266-9. DOI: 10.1089/sur.2011.015

15. Vorrasi J, Zinberg G. Concomitant Suppurative Parotitis and Condylar Osteomyelitis. J Oral Maxillofac Surg. 2016; 75(3): 543-9. https://www. ncbi.nlm.nih.gov/pmc/articles/PMC6099765/

16. Mohyud-din M, Haider S, Hameed A. Bilateral suppurative parotitis in a newborn. J Coll Physicians Surg Pak. 2006; 16(4): 301-2. https:// pubmed.ncbi.nlm.nih.gov/16624200/

17. Singh K. Bilateral parotid abscess in a neonate. Indian Pediatr. 2006; 43(11): 100910. https://www.researchgate.net/publication/239074285_Parotid_abscess_in_A_ late_premature_infant_A_case_report. doi: 10.1177/1742271X18766705

18. Zajkowski P, Choińska AO. Standards for the assessment of salivary glands-an update. J UItrason. 2016; 16(65): 175-90. https://www.ncbi. nlm.nih.gov/pmc/articles/PMC4954863/

19. Abdel Razek AAK, Mukherji S. Imaging of sialadenitis. Neuroradiol J. 2017; 30(3): 205-15. DOI: $10.1177 / 1971400916682752$

20. Wilson KF, Meier JD, Ward PD. Salivary gland disorders. Am Fam Physician. 2014; 89(11): 8828. https://pubmed.ncbi.nlm.nih.gov/25077394/

21. Al-Dajani N, Wootton SH. Cervical lymphadenitis, suppurative parotitis, thyroiditis, and infected cysts. Infect Dis Clin North Am. 2007;21(2): 523-41. DOI: 10.1016/j.idc.2007.03.004

22. Hernandez S, Busso C, Walvekar RR. Parotitis and sialendoscopy of the parotid gland. Otolaryngol Clin North Am. 2016; 49(2): 381-93. https://pubmed.ncbi.nlm.nih.gov/26912292/

Submetido em: 7-11-2019

Aceito em: 26-11-2020 